$\mathrm{CHO}$ cytosol were added back. The restorative activity of the purified NSF was inhibited by the monoclonal antibody (Fig. 2, inset). The molar ratio of pentameric antibody to NSF tetramers required to obtain half-maximal inhibition of the recovered fusogenic activity $(\sim 1.5)$ resembles the ratio reported to inhibit NSF activity in Golgi transport $(\sim 0.96)^{8}$.

NSF is highly sensitive to heat inactivation in the absence of ATP $^{8}$. We observed that the NEM-sensitive restorative activity for endocytosis of our cytosol preparations from both $\mathrm{CHO}$ and $\mathrm{J} 774$ cell lines remained stable at $37^{\circ} \mathrm{C}$ as long as they had not been gel-filtered to remove residual adenine nucleotides. Once the cytosols had been gel-filtered, the restorative NEM-sensitive fusogenic activity was lost after only a few minutes of incubation at $37^{\circ} \mathrm{C}$ (data not shown). ATP $(1 \mathrm{mM})$ stabilizes the factor, as has been observed for NSF within the context of Golgi transport ${ }^{8}$.

Our results indicate that the fusion among early endocytic vesicles which contain ligand internalized by receptor-mediated endocytosis requires NSF. Thus, our experiments clearly extend the scope of the activities of NSF beyond the secretory pathway. Exactly which step(s) and endocytic vesicles use NSF, or whether they all do, remains to be determined. Anti-NSF antibody has also an inhibitory effect on reconstitution assays where endocytic vesicles fuse with plasma membrane-derived vesicles ${ }^{6}$ or with protease containing compartments ${ }^{7}$ (data not shown).

We conclude that NSF plays an important part in fusion of endocytic vesicles during early stages of receptor-mediated endocytosis. NSF appears to require a set of both soluble and membrane proteins to associate with Golgi membranes ${ }^{11}$. It has not been determined whether these other components are also required for the activity of NSF in the endocytic pathway. NSF, nevertheless, appears to be a component of a machinery available to mammalian and other eukaryotic cells to catalyse the fusion of intracellular membranes. In addition to its requirement for Golgi transport, there is now evidence that vesicle transport from endoplasmic reticulum to Golgi also requires $N_{S F}{ }^{12}$. The ability of NSF to catalyse vesicle fusion in both secretory and endocytic pathways implies that the factor itself may not constitute a recognition signal for the regulation of vesicle fusion specificity. Other recognition signals must be present on the cytoplasmic surface of these vesicles that determine the fate of the transport vesicle.

Received 23 March; accepted 28 April 1989.

1. Diaz, R., Mayorga, L. \& Stahl, P. J. biol. Chem. 263, 6093-6100 (1988).

2. Braell. W.A. Proc. Natn. Acad. Sci. U.S.A. 84, 1137-1141 (1987).

3. Woodman, P.G. \& Warren, G. Eur. J. Biochem. 173, 101-108 (1988)

4. Glick, B.S. \& Rothman, J.E. Nature 326, 309-312 (1987).

5. Gruenberg, J.E. \& Howell. K.E. EMBO J. 5, 3091-3101 (1986)

6. Mayorga, L.S., Diaz, R. \& Stahl, P.D. L. biol. Chem. 263, 17213-17216 (1988).

7. Mayorga, L.S., Diaz, R. \& Stahl, P.D. J. biol. Chem. 264, 5392-5399 (1989).

8. Block, M.R., Glick, B.S., Wilcox, C.A., Wieland, F.T. \& Rothman, J.E. Proc. natn. Acad. Sci. U.S.A. 85, 7852-7856 (1988).

9. Malhotra V Orci, L Glick, B.S. Block, M.R. \& Rothman, JE. Cell 54, 221-227 (1988).

10. Wilson, D.W. et al. Nature 339, 355-359 (1989).

11. Weidman, P.J., Melancon, P., Block, M.R. \& Rothman, J.E. J. Cell Biol. 108, 1589-1596 (1988).

12. Beckers, C.J.M., Block, M.R., Glick, B.S., Rothman, J.E. \& Balch. W.E. Nature 339, 397-398 (1989).

ACKNOWLEDGEMENTS. We thank Lia E.B. Mayorga for technical assistance. The work was supported, in part, by the NIH. L.M. is a fellow of the National Research Council of Argentina. P.W. is a fellow of the NIH.

\title{
CORRIGENDUM
}

\section{psbA genes indicate common ancestry of prochlorophytes and chloroplasts}

\section{Clifford W. Morden \& Susan S. Golden}

Nature 337, 382-385 (1989)

THE phylogenetic tree shown in Fig. 3 of this letter was generated using a data set in which numeric coding errors have been found. These were detected by using a new version of PAUP ${ }^{1}$ which interprets the single-letter amino acid code directly and does not require coding. The uncoded amino acid data set was analysed as previously reported, using the branch and bound option of PAUP ${ }^{1}$. In the previous analysis many equally parsimonious trees were obtained when different treatments were used to accommodate the seven-amino-acid domain missing in Prochlorothrix hollandica and green chloroplast-containing taxa. In the current analysis only two phylogenetic tree topologies were obtained, corresponding to the published tree shown in Fig. 3 and a variation described in the text, in which $P$. hollandica was associated in a lineage including the Synechococcus sequences. The only variations observed were slight changes in branch lengths among the different treatments.

When the seven-amino-acid domain missing in some taxa was excluded from the analysis, or when its presence or absence was treated as a single binary character, a unique phylogenetic tree was generated associating the $P$. hollandica and Synechococcus sequences. Two methods were used to consider presence or absence of the domain as equivalent to two amino acid changes. If each character in the seven-amino-acid domain was weighted two-sevenths, one tree was obtained associating $P$. hollandica with Synechococcus. However, when the seven amino acids were treated as 'missing data' from the taxa that lack those residues, and an additional character having a weight of two was added to designate presence or absence of that domain, two trees were generated: one as described above and one like that shown in Fig. 3. Any additional significance ascribed to the seven-aminoacid domain, from treating it as the equivalent of three to seven amino acid changes, produced only the phylogenetic tree shown in Fig. 3.

This analysis showed that the distribution of the seven-aminoacid domain among taxa is the chief characteristic of the $p s b A$ genes, suggesting that $P$. hollandica is part of a lineage that led to chloroplasts after a divergence from the cyanobacteria. Phylogenetic analysis of the amino-acid sequences supports this relationship only if the presence or absence of residues in this domain is considered to be more significant than one or two amino-acid substitutions.

1. Swofford. D. L. PAUP version 3.0 ( $\beta$-test version, unpublished) 